\title{
Ground Performance Measurements of the Glory Aerosol Polarimetry Sensor
}

\author{
Steven Persh $^{\mathrm{a}}$, Y.J. Shaham ${ }^{\mathrm{a},{ }^{*}}$, Oren Benami ${ }^{\mathrm{a}}$, Brian Cairns ${ }^{\mathrm{b}}$, Michael I. Mishchenko ${ }^{\mathrm{b}}$, \\ Jeffrey D. Hein ${ }^{c}$, Bryan A. Fafaul ${ }^{c}$ \\ ${ }^{a}$ Raytheon Space and Airborne Systems \\ ${ }^{\mathrm{b}}$ NASA Goddard Institute for Space Studies \\ ${ }^{\mathrm{c}}$ NASA Goddard Space Flight Center, Greenbelt, Md
}

\begin{abstract}
The Aerosol Polarimetry Sensor (APS) is the primary Earth observing instrument of the Glory Mission. It is expected to launch into space in the $4^{\text {th }}$ quarter of 2010. This paper summarizes results from the APS ground-testing, completed in 2009. Ground testing established that the instrument meets or exceeds performance requirements: SNR, dynamic range, radiometric accuracy, polarimetric accuracy, response vs. scan angle, boresight co-alignment, and calibration sources accuracy. The APS demonstrated excellent performance stability during sensor and spacecraft level testing over a wide range of environmental conditions. The APS will be a significant improvement over existing sensors that measure aerosols from space. It will provide the scientific community with new information about the distribution and properties of aerosols around the globe. Scientists will use APS data to estimate the radiative forcing imposed on the Earth by aerosols, to assess the effects of aerosols on the Earth's climate.
\end{abstract}

Keywords: Polarization, polarimetry, global warming, aerosols, Glory Mission, Aerosol Polarimetry Sensor

\section{Introduction}

The Aerosol Polarimetry Sensor (APS) is the primary Earth observing instrument of the Glory Mission ${ }^{1}$. It was developed by Raytheon SAS under the Glory Program Office, GSFC 426, with scientific support from the Goddard Institute for Space Studies, GSFC 611. The APS ${ }^{2}$ will provide the scientific community with a significant improvement over existing sensors measuring aerosols from space ${ }^{3}$. The upcoming launch of this highly accurate polarimetric sensor will substantially enhance the scientific community's knowledge of the distribution and properties of aerosols around the globe.

The Glory satellite will be placed in a $705 \mathrm{~km}$ sun-synchronous orbit at 98.2 degrees inclination. The planned location on orbit is the NASA afternoon orbit less than two minutes after the CALIPSO satellite with the Aqua satellite a further minute ahead. The APS sensor observes each scene from 248 unvignetted viewing angles along track at an angular range of $+50 /-65^{\circ}$. It will be launched on a Taurus XL (3110) Launch Vehicle.

The APS finished ground testing in mid 2009 and is expected to launch in November 2010. This paper focuses on the ground testing of APS at Raytheon. Radiometric, polarimetric, spatial, temporal and spectral characteristics of the instrument were measured and trended. Radiometric tests included SNR, dynamic range, linearity, and radiometric accuracy. Polarimetric tests included polarization accuracy and stability. Additional tests included response vs. scan angle, boresight co-alignment and calibration source accuracy. Many of the APS performance tests were repeated multiple times over a wide range of environments during sensor and spacecraft level testing, and the APS demonstrated excellent stability throughout these tests.

\footnotetext{
*JeffShaham@raytheon.com, 310-647-4524
} 


\section{The APS Instrument}

The APS instrument without its multilayer insulation blankets is shown in Figure 1. The APS instrument with its multilayer insulation blankets, and mounted on the bus is shown in Figure 2. Its optical system consists of six boresighted telescopes ( 3 pairs) oriented in a radial bunch, 4 of which pass visible and near-infrared (VNIR) bands, and 2 of which pass short-wave infrared (SWIR) bands. Each pair of telescopes covers the same three bands; therefore the entire system has a total of 9 bands ( 3 telescopes pairs $x 3$ bands) at the following wavelengths: 412, 443, 555, 673, 865, $910,1378,1610$ and $2250 \mathrm{~nm}$. The bands are numbered sequentially from the shortest wavelength to the longest wavelength (e.g. band 1 is $412 \mathrm{~nm}$ ). Wollaston prisms are located behind the telescopes and serve as polarization analyzers for the system. Three of the six telescopes have the Wollaston prisms oriented to resolve the $0^{\circ} / 90^{\circ}$ (Channel 1 / Channel 2) polarization components while the other three conjugate telescopes have the Wollaston prisms physically rotated by $45^{\circ}$ in azimuth with respect to the first set to resolve the $45^{\circ} / 135^{\circ}$ (Channel $4 /$ Channel 3) components, resulting in a total of 36 measurements, with one detector for each measurement. The VNIR bands utilize blue and red enhanced silicon photo-diodes. The SWIR energy is folded into a cryogenic Dewar and relayed by field lenses to form a pupil image on mercury cadmium telluride detectors, in order to minimize detector spatial response variations. The FPA data is linearly transformed to the first 3 Stokes parameters (I, Q, U) with one redundant measurement that allows the Stokes parameters to be determined, if any one channel in each band fails.

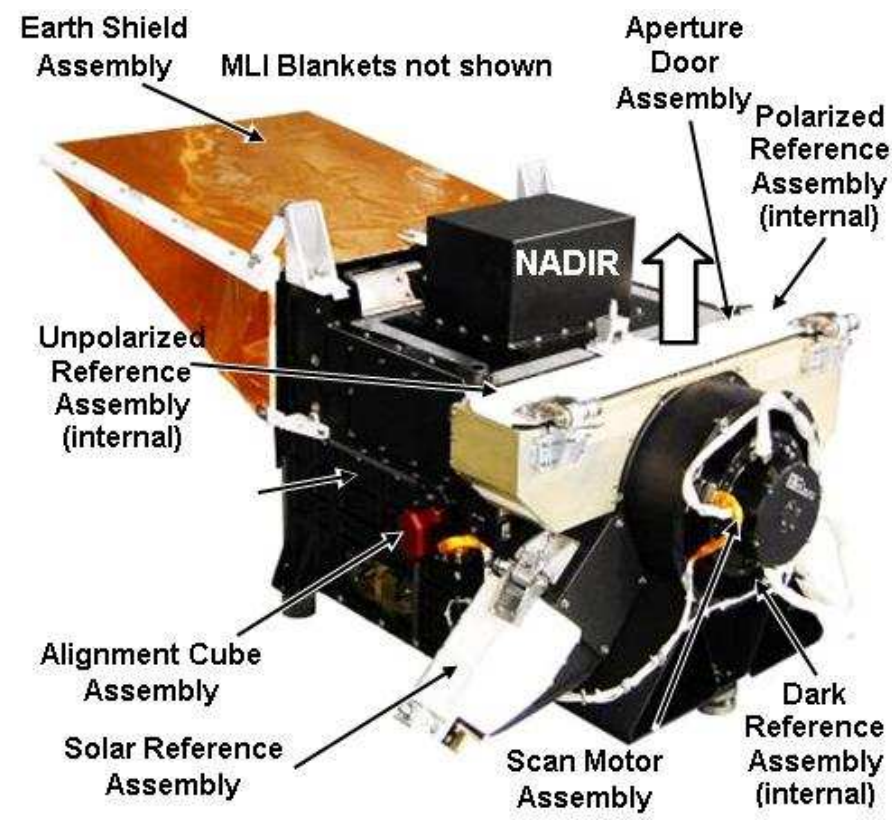

Figure 1: APS Instrument

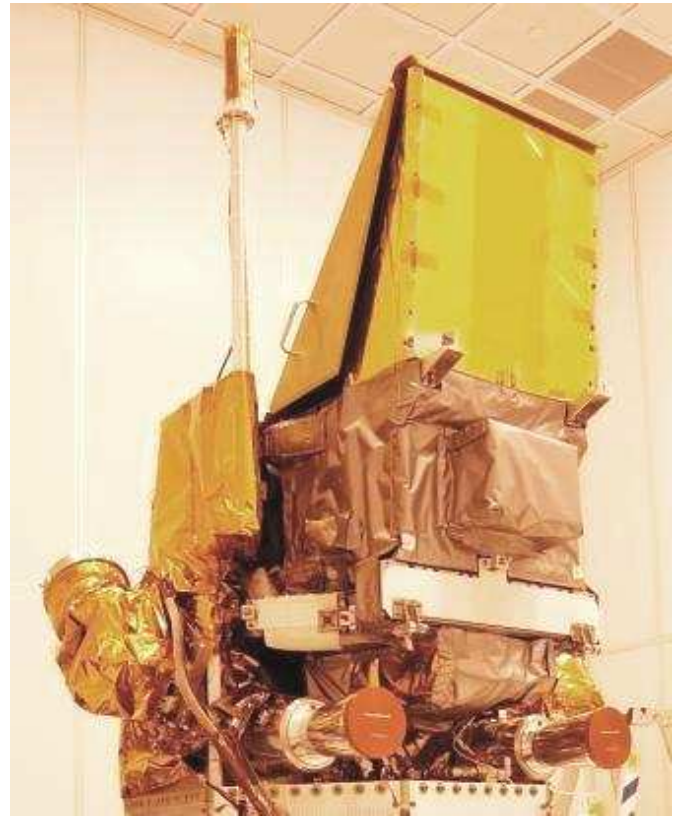

Figure 2: APS Instrument Mounted on Bus

\section{Testing Overview}

The instrument verification tests can be categorized into three areas: functional, performance, and environmental tests. The functional tests include the following:

$\circ$ Grounding and shielding tests

- Power tests: power, survival power, voltage, current, regulation, ripple and transients

- Mode tests: Off, Safe, Operational and Survival modes

○ Telemetry: Commands and Data handling

- Scanning characteristics

- Mechanical characteristics (size, weight, and center of gravity)

The environmental test conditions include launch vibrations, satellite EMI and thermal tests. The APS met or exceeded the functional and environmental requirements as well as the performance tests. This paper focuses on the results of the performance tests, including performance over the required temperature ranges. 


\section{Summary of Measured Performance}

The APS requirements and the predicted performance of the design was first published in $2007^{2,3}$. These performance values were subsequently confirmed via ground testing (Table 1), and the margins were found to be larger than predicted, in most cases.

Table 1: Ground Test Performance Summary

\begin{tabular}{|l|l|l|}
\hline Parameter & Requirement & Ground Performance \\
\hline Dynamic Range (SWIR) & 10 to 65,536 counts & 17 to 56,138 counts \\
\hline Dynamic Range (VNIR) & 100 to 65,536 counts & 127 to 56,138 counts \\
\hline SNR & 94 to 235 (varies by band) & 288 to 792 (varies by band and channel) \\
\hline Radiometric Accuracy @ min radiance & $5.1 \%$ to $17.5 \%$ (varies by band) & $2.5 \%$ to $5.2 \%$ (varies by band) \\
\hline Spectral Response & See Table 3 & See Table 3 \\
\hline Relative Spectral Accuracy & $1.3 \%$ to 3\% & $0.8 \%$ to $2.6 \%$ \\
\hline Wollaston Prisms Absolute Orientation Error & $<0.1$ degrees & $<0.025$ degrees \\
\hline Wollaston Prisms Relative Orientation Error & $<0.05$ degrees & $<0.018$ degrees \\
\hline Polarimetric Accuracy & $+/-0.2 \%$ (for degree of polarization $<20 \%)$ & $<0.14 \%$ \\
\hline Bore-sight co-alignment for a given polarization & $<0.4 \mathrm{mrad}$ & $0.3 \mathrm{mrad}$ @ ambient \\
\hline Bore-sight co-alignment for both polarizations & $<0.6 \mathrm{mrad}$ & $0.5 \mathrm{mrad} @$ ambient \\
\hline IFOV & $+/-0.4 \mathrm{mrad}$ & $+/-0.16 \mathrm{mrad}$ \\
\hline Scene Matching & $+/-0.5 \%$ & $<0.05 \%($ VNIR), $<0.25 \%$ (SWIR) \\
\hline Radiometric Error vs. Scan Angle & $<1 \%$ & $<0.69 \%$ \\
\hline Polarimetric Error vs. Scan Angle & $<0.2 \%$ & $<0.1 \%$ \\
\hline Unpolarized Reference Assembly DoLP & $<0.05$ & $<0.008$ \\
\hline Polarized Reference Assembly DoLP & $>0.99$ & $>0.999$ \\
\hline Dark Reference Assembly \% of Scene Radiance & $<0.05$ & $<0.03$ \\
\hline
\end{tabular}

All the polarimetric, radiometric, and spatial (IFOV matching) requirements were met and were consistent over the environmental test ranges. Overall, the instrument performed much better than required and maintained excellent performance throughout environmental testing, with only one exception; the telescopes co-alignment was not compliant at cold acceptance temperature. The sources of the misalignment have been determined and a concept of operations that compensates for this issue will be used on orbit.

A waiver for telescope bore-sight co-alignment was granted based on subsequent modifications to the thermal balance of the APS via modifications to the EM radiator, a plan for continuous use of the Optical Detection Module (ODM) trim heater on orbit, and a detailed analysis of the impact on the NASA level 2 requirements. The conclusion being that at an operating temperature of $1.5^{\circ} \mathrm{C}$ (required to meet level 2 requirements on orbit) is attainable with some considerable margin.

Sensor performance was determined under ambient, cold, and hot conditions. Relative and absolute accuracy values were determined. Performance tests were conducted before, during and after the environmental tests. The values over the multiple tests period form trends. The trends for all measures showed consistent compliant results, with small (< $10 \%$ ) variations relative to the baseline ambient values.

\section{Ground Test Results}

This section discusses many of the APS ground test results: radiometric performance, polarimetric performance, FOV and IFOV, boresight co-alignment, and response vs. scan angle.

\subsection{Dynamic Range}

For this sensor, the dynamic range should make maximum use of the available 16 bit dynamic range of the ADC while providing appropriate overhead margin against the maximum predicted radiance level and ensuring that the minimum radiance levels are well differentiated from the dark values (greater than 10 for the SWIR Bands and greater than 100 for the VNIR Bands). The signals for all bands and all polarization channels fall within this dynamic range (Figure 2). The SWIR data at the minimum dynamic range (not shown here) varied from 15 to 40 counts. 

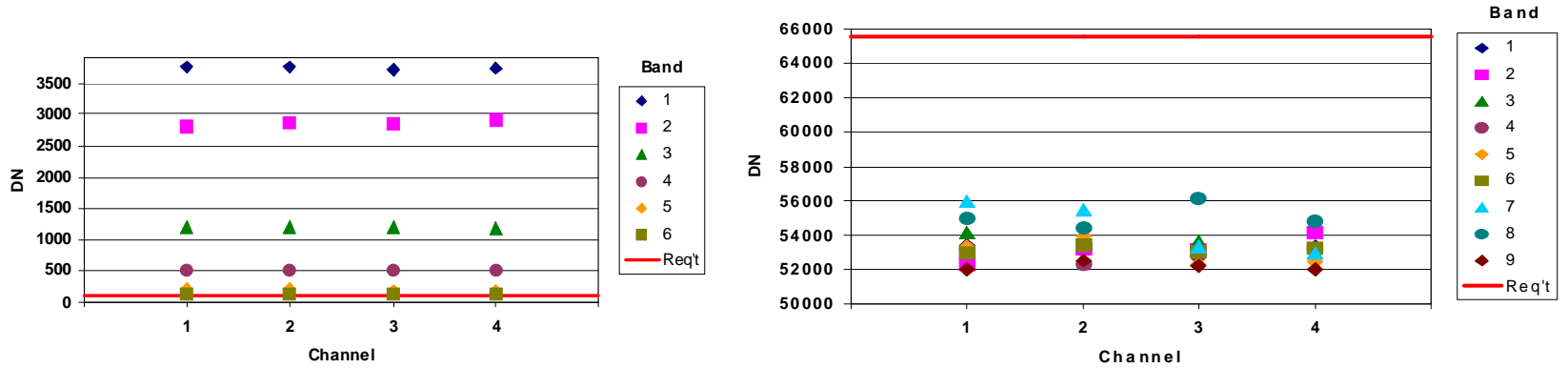

\subsection{SNR}

Figure 2: Minimum (left) and Maximum (right) digital counts

SNR was evaluated for all bands at baseline ambient conditions. SNR performance was better than required, for all 4 polarization channels, at all 9 wavelengths (Figure 3).

The spherical integrating source (SIS) ${ }^{4}$ used for testing the instrument could not provide the specified.typical radiance levels in all bands. Therefore, the signal and shot noise from the measured radiance was scaled to the radiance at which the SNR requirement was specified, in order to arrive at the SNR values shown in Figure 3, for each Band and Channel. The fixed noise terms were separated out prior to scaling SNR, and then recombined with the scaled SNRs, to get the numbers shown in Figure 3.

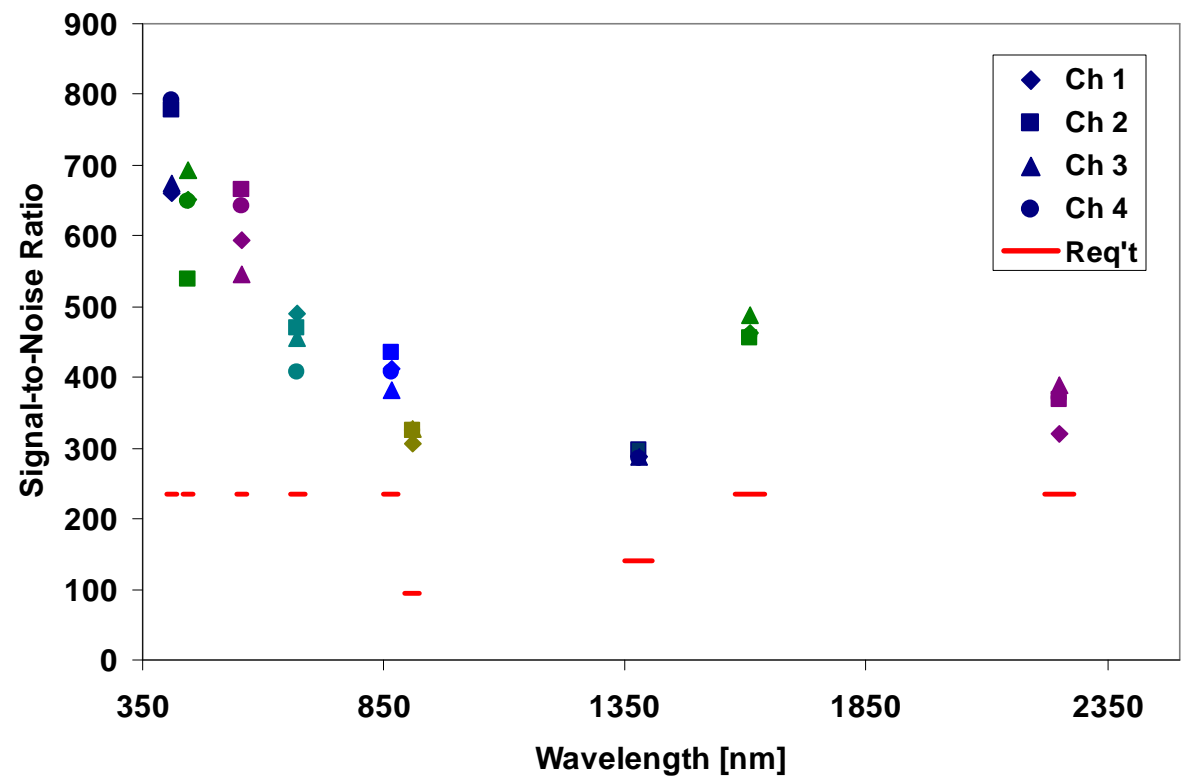

Figure 3: SNR exceeded requirements (red dashes)

\subsection{Dynamic Range and SNR Trending}

The dynamic range and SNR were repeatedly evaluated throughout the testing period, starting with the baseline ambient tests, and before, during and after the thermal vacuum tests. The VNIR bands were consistent within 5\%, and the SWIR bands were consistent within $9 \%$. The larger variability in the SWIR bands was caused by different behavior in thermal vacuum tests compared with the ambient tests. This was the result of a small vacuum leak in the SWIR dewar-detector assembly, that was discovered during the ambient testing, and which reduced the transmission to the SWIR detectors. This will have no impact on the on-orbit performance of the APS. 
The SNR trending for SWIR bands (Figure 4) shows SNR values higher than the ambient baseline for bands 8 and 9, and worse than the ambient baseline for band 7; especially channel 4 (7-4). Since the radiometric and polarimetric values can be determined from 3 channels, the noise in channel 4 does not affect band 7 accuracies significantly.

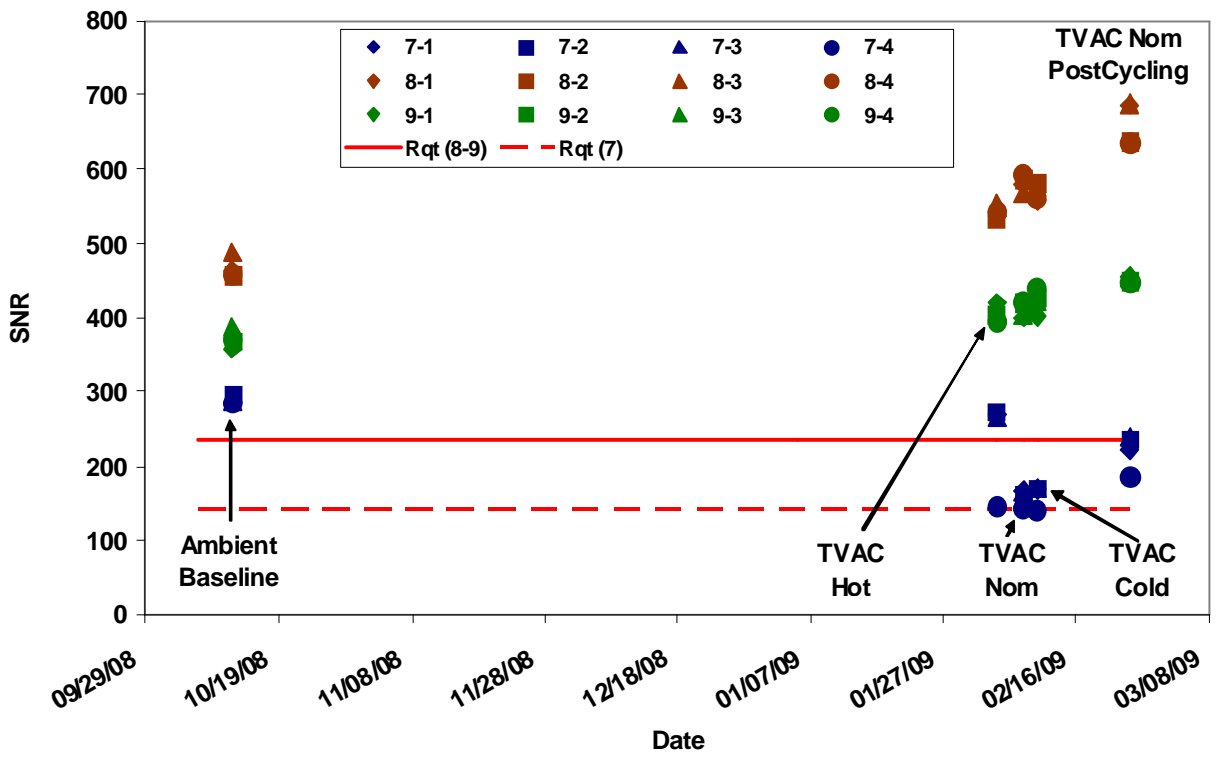

\subsection{Radiometric accuracy}

Figure 4: SNR trending for SWIR bands

Ground measurements of sensor radiometric accuracy (Table 2) have three main error contributors: radiance of the spherical integrating source (SIS), transfer of this radiance to the sensor, and sensor errors. The SIS calibration error was the largest contributor to the radiometric accuracy error (2.2\% to $4 \%$, when referenced to the NIST standard). The range of calibration accuracy errors for all the bands, at the minimum radiance level, was $2.5 \%$ to $5.2 \%$.

Table 2: APS Radiometric accuracy (\%) for each band at the minimum radiance level

\section{Ground, Radiance-based Absolute Calibration Accuracy - Lmin}

\begin{tabular}{|c|c|c|c|c|c|c|c|c|c|}
\hline & $412 \mathrm{~nm}$ & $443 \mathrm{~nm}$ & $555 \mathrm{~nm}$ & $\begin{array}{c}672 \mathrm{~nm} \\
\text { analysis }\end{array}$ & $\begin{array}{c}\mathbf{8 6 5} \mathbf{~ n m} \\
\text { analysis }\end{array}$ & $\begin{array}{l}910 \mathrm{~nm} \\
\text { analysis }\end{array}$ & $\begin{array}{c}1378 \mathrm{~nm} \\
\text { analysis }\end{array}$ & $\begin{array}{c}1610 \mathrm{~nm} \\
\text { analysis }\end{array}$ & $\begin{array}{c}2250 \mathbf{n m} \\
\text { analysis }\end{array}$ \\
\hline $\begin{array}{l}\text { STE Characterization } \\
\text { SIS Calibration Error } \\
\text { Additional Uncertainty for Uncalibrated Levels } \\
\text { Remove and Replace Upon Transport } \\
\end{array}$ & \begin{tabular}{r|}
2.5 \\
2.18 \\
0.80 \\
0.80 \\
\end{tabular} & \begin{tabular}{r|}
2.5 \\
2.18 \\
0.80 \\
0.80 \\
\end{tabular} & \begin{tabular}{r|}
2.5 \\
2.18 \\
0.80 \\
0.80 \\
\end{tabular} & $\begin{array}{r}2.5 \\
2.22 \\
0.80 \\
0.80 \\
\end{array}$ & \begin{tabular}{r|}
2.5 \\
2.25 \\
0.80 \\
0.80 \\
\end{tabular} & $\begin{array}{r}2.7 \\
2.45 \\
0.80 \\
0.80 \\
\end{array}$ & \begin{tabular}{r|}
4.1 \\
3.98 \\
0.80 \\
0.80 \\
\end{tabular} & \begin{tabular}{r|}
4.1 \\
3.93 \\
0.80 \\
0.80 \\
\end{tabular} & $\begin{array}{l}4.0 \\
4.00\end{array}$ \\
\hline $\begin{array}{l}\text { Calibration Transfer (SIS to Sensor) } \\
\text { Stray Light } \\
\text { Atmospheric Absorption } \\
\text { Residual SIS Polarization } \\
\end{array}$ & \begin{tabular}{r|}
0.1 \\
0.08 \\
0.07 \\
\end{tabular} & \begin{tabular}{r|}
0.1 \\
0.10 \\
0.04 \\
\end{tabular} & \begin{tabular}{r|}
0.1 \\
0.05 \\
0.10 \\
\end{tabular} & $\begin{array}{r}0.1 \\
0.09 \\
0.03 \\
\end{array}$ & $\begin{array}{r}0.1 \\
0.06 \\
0.04 \\
\end{array}$ & $\begin{array}{r}0.1 \\
0.09 \\
0.10 \\
0.03 \\
\end{array}$ & \begin{tabular}{r|}
1.0 \\
0.04 \\
1.00 \\
0.05 \\
\end{tabular} & \begin{tabular}{r|}
0.1 \\
0.04 \\
0.10 \\
0.06 \\
\end{tabular} & $\begin{aligned} 0.2 \\
0.04 \\
0.16 \\
\end{aligned}$ \\
\hline $\begin{array}{l}\text { Sensor Errors } \\
\text { Measured Sensor Error at Lmin } \\
\text { Measured Sensor Error at Lmax (for analysis) } \\
\text { APS Non-linearity (if testing not made at Ltyp) } \\
\text { EM Microlinearity (for radiances below } 3000 \mathrm{DN} \text { ) } \\
\text { Response Variation with Scan Angle } \\
\text { Out-of-Band (OOB) Response } \\
\end{array}$ & $\begin{array}{l}\mathbf{0 . 6} \\
0.52\end{array}$ & $\begin{array}{l}\mathbf{0 . 6} \\
0.10\end{array}$ & $\begin{array}{l}1.3 \\
1.21\end{array}$ & $\begin{array}{l}1.0 \\
0.78 \\
0.02 \\
0.10 \\
0.64 \\
0.20\end{array}$ & $\begin{array}{l}1.03 \\
0.03 \\
0.25 \\
0.48 \\
0.24\end{array}$ & $\begin{array}{l}0.23 \\
0.02 \\
0.42 \\
0.66 \\
0.34\end{array}$ & \begin{tabular}{l|}
2.5 \\
0.37 \\
0.02 \\
2.43 \\
0.44 \\
0.33
\end{tabular} & \begin{tabular}{r|}
2.1 \\
1.48 \\
0.02 \\
1.41 \\
0.28 \\
0.43
\end{tabular} & $\begin{array}{l}3.3 \\
0.88 \\
0.04 \\
3.09 \\
0.41 \\
0.48\end{array}$ \\
\hline TOTAL ERROR & 2.5 & 2.5 & 2.8 & 2.7 & 2.8 & 2.8 & 4.9 & 4.6 & 5.2 \\
\hline
\end{tabular}

The variation of radiometric accuracy with test radiance levels for band 4, telescope 1, is shown in Figure 5. Verification was determined by a combination of test and analysis because of SIS uncertainties at the lower end of dynamic range. For bands which could not be tested at or below the typical radiance $\mathrm{L}_{\mathrm{typ}}$, measured sensor error at $\mathrm{L}_{\max }$ was extrapolated and error terms were added to account for the measured non-linearity at the low end of the dynamic range. 


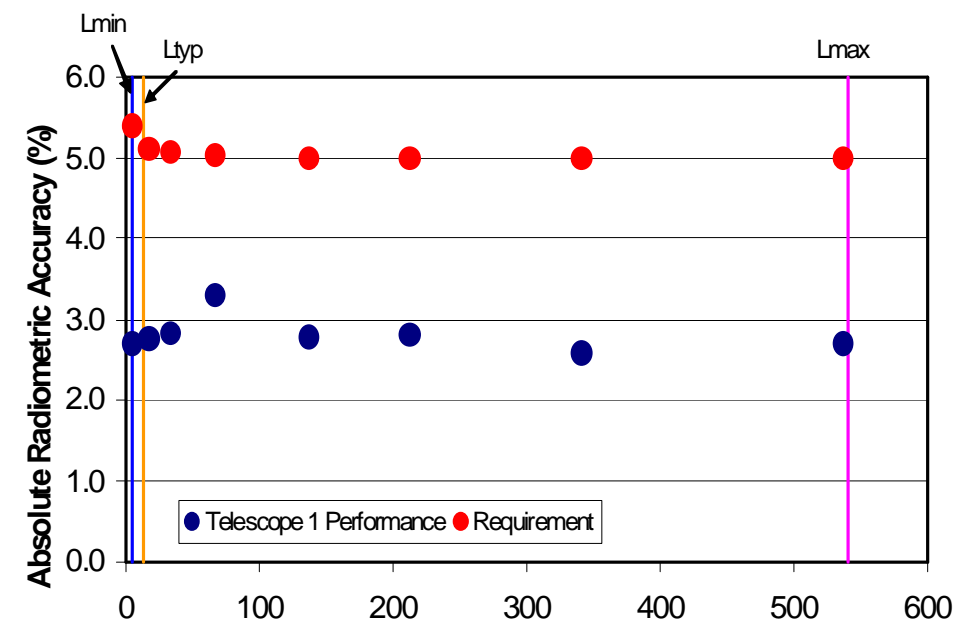

Figure 5: Variation of Radiometric Accuracy (\%) with Test Radiance (W/m^2/micrometer/sr) for Band 4, Telescope 1

\subsection{Spectral Response and Relative Spectral Accuracy}

The spectral response of the instrument is based on analysis of filter and dichroic beamsplitter component data and the spectral responsivity of the detectors. Each vendor provided the spectral response function for the components that they supplied. The product of the spectral response (e.g. transmission) of each component in the optical path including the detectors is the system spectral response. For each band, Table 3 provides the center wavelength, the full width at half maximum, and the out-of-band percent response. For all bands and considering thermal shifts, the values are within the requirements.

Table 3: Sensor Spectral Response

\begin{tabular}{|c|c|c|c|c|c|c|}
\hline & Requirement & Performance & Requirement & Performance & Requirement & Performance \\
\hline Nominal Band (nm) & Center Wavelength (nm) & Center Wavelength (nm) & FWHM (nm) & FWHM (nm) & OOB (\%) & OOB (\%) \\
\hline 412 & $412+/-2$ & 413 & $20+/-2$ & 18.2 & 1 & 0.3 \\
\hline 443 & $443+/-3$ & 443 & $20+/-2$ & 18.9 & 1 & 0.3 \\
\hline 555 & $555+/-4$ & 555 & $20+/-3$ & 20.1 & 0.7 & 0.3 \\
\hline 672 & $672+/-5$ & 674 & $20+/-3$ & 20.7 & 0.7 & 0.2 \\
\hline 865 & $865+/-5$ & 866 & $39+/-5$ & 40.3 & 0.7 & 0.2 \\
\hline 910 & $910+/-5$ & 911 & $20+/-5$ & 19.2 & 1 & 0.3 \\
\hline 1378 & $1378+/-5$ & 1374 & $35+/-5$ & 36.5 & 1 & 0.3 \\
\hline 1610 & $1610+/-14$ & 1603 & $60+/-9$ & 52.5 & 0.7 & 0.4 \\
\hline 2250 & $2250+/-13$ & 2259 & $75+/-6$ & 79 & 1 & 0.5 \\
\hline
\end{tabular}

The relative spectral accuracy is the variation in radiance between adjacent bands. For example band 1 to band 2 relative spectral accuracy was $<0.9 \%$, compared to a requirement of $<1.3 \%$. The analysis showed that the relative spectral accuracy was better than required for all adjacent bands in the APS system.

\subsection{Polarimetric Accuracy}

The normalized Stokes components $\mathrm{q}$ and $\mathrm{u}$ were obtained from in processing the test data (Figure 6) ${ }^{2,5}$. The absolute and relative orientation and uncertainty of the Wollaston prisms are given in Table 4. The uncertainties were much better than the required absolute uncertainty error ( 0.1 degrees) and relative uncertainty error ( 0.05 degrees $)$. 


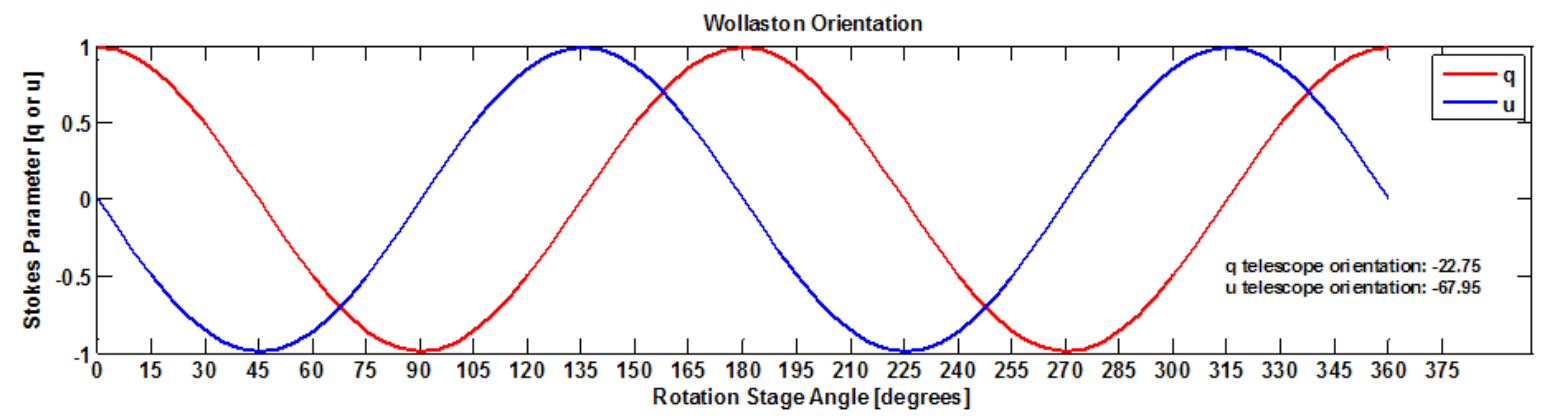

Figure 6: Sinusoids produced the normalized Stokes components (q and u)

Table 4: Wollaston Prisms absolute and relative uncertainty

\begin{tabular}{|c|c|c|c|c|}
\hline Telescope & $\begin{array}{c}\text { Absolute Orientation } \\
\text { (deg) }\end{array}$ & $\begin{array}{c}\text { Absolute Uncertainty } \\
\text { (deg) }\end{array}$ & $\begin{array}{c}\text { Relative Clocking Error } \\
\text { (deg) }\end{array}$ & $\begin{array}{c}\text { Uncertainty of Relative Clocking Error } \\
\text { (deg) }\end{array}$ \\
\hline VNA1 & -22.754 & 0.024 & \multirow{2}{*}{0.19} & \multirow{2}{*}{0.018} \\
\hline VNA2 & -67.947 & 0.024 & & \\
\hline VNB1 & -23.166 & 0.025 & \multirow{2}{*}{-0.405} & \multirow{2}{*}{0.015} \\
\hline VNB2 & -67.761 & 0.025 & & \\
\hline SW1 & -22.824 & 0.024 & \multirow{2}{*}{0.181} & \multirow{2}{*}{0.014} \\
\hline SW2 & -68.006 & 0.024 & & \\
\hline
\end{tabular}

The polarization accuracy trending error (Figure 7) was evaluated starting with the ambient tests, and continuing through and before and after the environmental tests over a period of 5 months. There were minimal changes in the trends, except for band 1, where stray light in TVAC testing increased the error. The magnitude of the stray light (reflection between STE and the chamber) induced error was characterized, and when corrected, it was found that there was a negligible difference between TVAC and ambient tests.

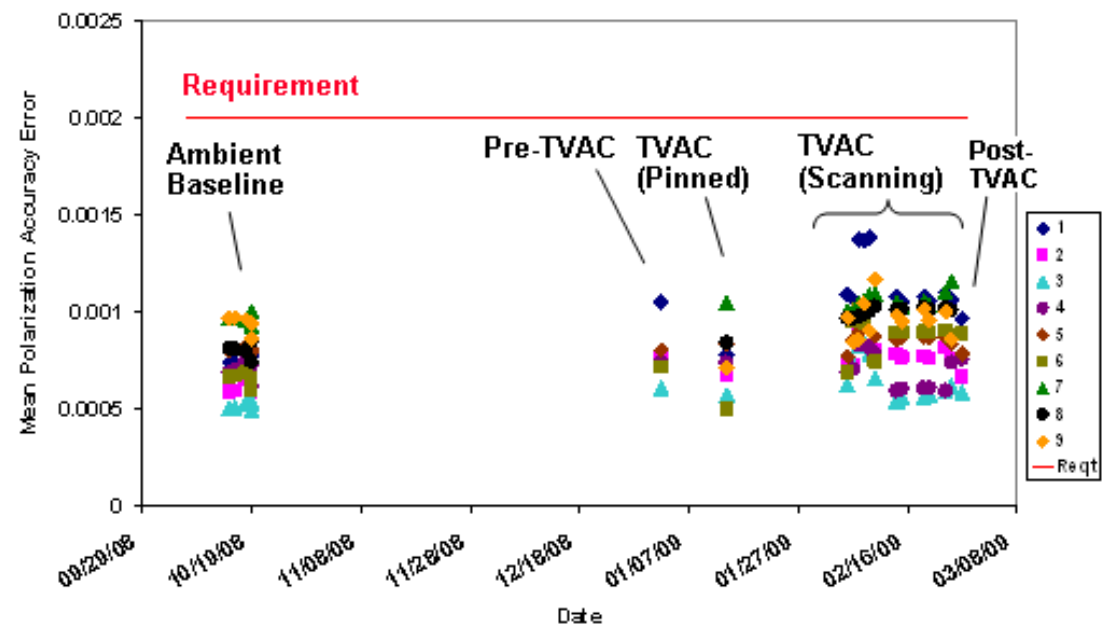

Figure 7: Polarization accuracy trend over the testing period

\subsection{Boresight co-alignment, IFOV, and Scene Matching}

In order to get good measurements, it is essential that all detected signals come from the same direction- i.e. their line of sight should be nearly identical. The boresight co-alignment requirements were $0.4 \mathrm{mrad}$ ( $5 \%$ error) for a given polarization and $0.6 \mathrm{mrad}$ ( $7 \%$ error) for all polarizations. 
The alignment accuracy test results at ambient temperature are presented in Figure 8. The direction of each of the 36 channels is indicated by a symbol. The lower right circle represents the boundary of the q polarization IFOV directions. The upper left circle represents the boundary of the u polarization IFOV directions. Each polarization falls within the 0.4 mrad requirement and both polarizations ( $\mathrm{q}$ and $\mathrm{u}$ ) fall within the $0.6 \mathrm{mrad}$ (large circle) requirement.

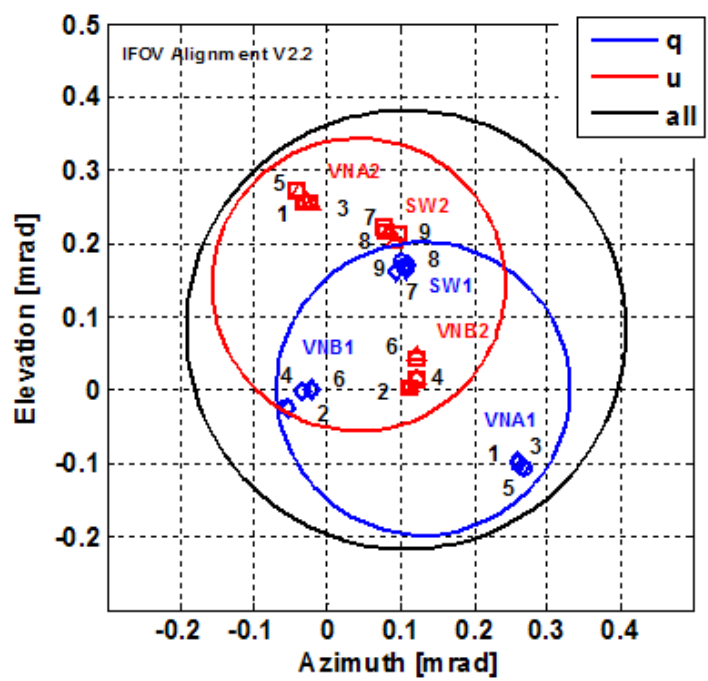

Figure 8: Telescope alignment- the symbols indicate the relative direction of the 36 channels

The co-alignment did not hold at cold temperatures- the large circle expanded to $1 \mathrm{mrad}$. The shifts are correlated with the Optical Detection Module (ODM) and the Scan Mirror Housing Assembly (SMHA) temperatures, with the SMHA having the stronger effect. The average shift with SMHA temperature change was 10 microradians $/{ }^{\circ} \mathrm{C}$, and the average shift with ODM temperature change was 3 microradians $/{ }^{\circ} \mathrm{C}$. Based on this analysis of the effects of temperature, the APS SMHA and ODM heaters will be used on orbit to bias the operating temperature to be as close to an ambient temperature of $20^{\circ} \mathrm{C}$ as feasible.

The IFOV size is determined in the same test that measures the boresight co-alignment, evaluating the azimuth and elevation FWHM, rather than the center of the response. Its accuracy is well within the tolerance requirement, with a maximum deviation of $0.16 \mathrm{mrad}$ from the $8 \mathrm{mrad}$ specification, and in most cases much smaller. The trend changes were insignificant.

The "Scene Matching" requirement ensures that the total response difference between the two detectors measuring the two orthogonal polarizations in each band is less than $0.5 \%$. The "Scene Matching" test showed that the two polarizations in the visible bands matched to an order of magnitude higher accuracy $(0.05 \%)$ for the VNIR bands, and a factor of 2 better $(0.25 \%)$ for the SWIR channels. This requirement is imposed in order to ensure that in the presence of a worst-case spatial heterogeneity (black and white checker-board similar to fair-weather cumulus over the ocean) within the IFOV, the polarization error is maintained at a low level (less than half the IFOV matching error based on a CauchySchwarz inequality). The test results indicate that errors caused by scene heterogeneity will be negligible.

\subsection{Radiometric Response vs. Scan Angle}

The APS was mounted on a rotary table, and radiometric data was taken at 10 scan angles spanning the scan field-ofview, at ambient only. A polarization scrambler was placed in the optical path to get unpolarized irradiance. The maximum deviation from the mean intensity coefficients (I1, I2) and gain coefficients (K1, K2 ${ }^{2}$ were well within the $1 \%$ requirement (Table 5). The larger deviations in the intensity coefficients are caused by fluctuations in the output of the SIS. The magnitude of variations indicates that even with this amplification of variability, the response vs. scan angle performance of the APS is outstanding. 
Table 5: Radiometric error response (maximum) deviation from the mean

\begin{tabular}{|c|c|c|c|c|c|}
\hline \multirow{2}{*}{ Band } & \multirow{2}{*}{ Req't } & \multicolumn{2}{|c|}{ Telescope \#1 } & \multicolumn{2}{c|}{ Telescope \#2 } \\
\cline { 3 - 6 } & & $\mathbf{K 1}(\%)$ & $\mathbf{I 1}(\%)$ & $\mathbf{K 2}(\%)$ & $\mathbf{I 2}(\%)$ \\
\hline $\mathbf{1}$ & $\mathbf{1 \%}$ & 0.27 & 0.24 & 0.29 & 0.23 \\
\hline $\mathbf{2}$ & $\mathbf{1 \%}$ & 0.03 & 0.51 & 0.05 & 0.46 \\
\hline $\mathbf{3}$ & $\mathbf{1 \%}$ & 0.07 & 0.35 & 0.05 & 0.55 \\
\hline $\mathbf{4}$ & $\mathbf{1 \%}$ & 0.05 & 0.64 & 0.04 & 0.61 \\
\hline $\mathbf{5}$ & $\mathbf{1 \%}$ & 0.06 & 0.48 & 0.03 & 0.63 \\
\hline $\mathbf{6}$ & $\mathbf{1 \%}$ & 0.04 & 0.66 & 0.03 & 0.69 \\
\hline $\mathbf{7}$ & $\mathbf{1 \%}$ & 0.08 & 0.44 & 0.05 & 0.71 \\
\hline $\mathbf{8}$ & $\mathbf{1 \%}$ & 0.07 & 0.28 & 0.10 & 0.54 \\
\hline $\mathbf{9}$ & $\mathbf{1 \%}$ & 0.05 & 0.41 & 0.06 & 0.62 \\
\hline
\end{tabular}

\subsection{Polarimetric Response vs. Scan Angle}

The polarization accuracy data over the scan angles was taken in the same way as the radiometric data, except that a wire grid polarizer was placed in the optical path instead of the polarization scrambler. The variation of the polarization error over the scan angles was less than $0.2 \%$, for an input polarization of $100 \%$. The variation in the degree of linear polarization (DoLP) is shown in Table 6, in percent.

Table 6: Variation of DoLP relative error (percent) with scan angle

\begin{tabular}{|c|c|c|}
\hline Band & Req't & DOLP Rel. Error \\
\hline $\mathbf{1}$ & $\mathbf{1} \%$ & 0.08 \\
\hline $\mathbf{2}$ & $\mathbf{1} \%$ & 0.11 \\
\hline $\mathbf{3}$ & $\mathbf{1} \%$ & 0.08 \\
\hline $\mathbf{4}$ & $\mathbf{1} \%$ & 0.10 \\
\hline $\mathbf{5}$ & $\mathbf{1} \%$ & 0.11 \\
\hline $\mathbf{6}$ & $\mathbf{1} \%$ & 0.09 \\
\hline $\mathbf{7}$ & $\mathbf{1} \%$ & 0.15 \\
\hline $\mathbf{8}$ & $\mathbf{1} \%$ & 0.12 \\
\hline $\mathbf{9}$ & $\mathbf{1} \%$ & 0.14 \\
\hline
\end{tabular}

\section{Calibration Source Accuracy}

The APS instrument will be monitored on orbit using calibrators, to ensure that the calibration limits are maintained over the spacecraft lifetime. The APS includes four calibration assemblies for on-orbit calibration: the Unpolarized Reference Assembly (URA), the Polarized Reference Assembly (PRA), the Dark Reference Assembly (DRA), and the Solar Reference Assembly (SRA) (Figure 9). The first three use reflected Earth radiance (or its absence in the case of the DRA) as a their own radiance source of illumination, while the SRA relies on the Sun as a source. The accuracies of the calibration sources were determined in ground tests.

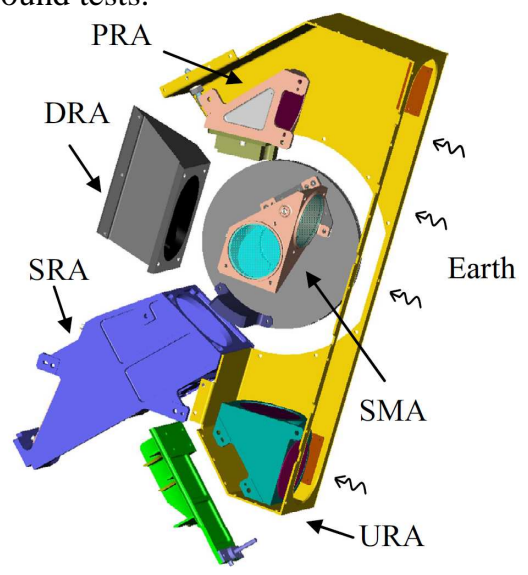

Figure 9: Calibration sources 


\subsection{Unpolarized Reference Assembly}

The Unpolarized Reference Assembly (URA) provides intra-telescope and inter-telescope relative responsivity factors, which are critical to meet polarization accuracy and stability. The URA is nadir facing so that the spectral content and dynamic range of its depolarized scene look is virtually the same as that observed in the scene polarization measurement samples. It provides a depolarized view for all of the detectors by means of a polarization scrambler composed of two wedges of crystalline quartz with the optical axes orientated $45^{\circ}$ to each other - a spatially varying retardance is created which scrambles any scene polarization.

The URA accuracy was measured following the radiometric and calibration tests of the instrument. URA signal data was obtained with a polarized STE source and non-polarized STE sources. The unpolarized signals provided data to calculate the relative gain coefficients $\mathrm{K} 1$ and $\mathrm{K} 2$. The polarized signal was generated with the wire grid polarizer inserted into the SIS optical path and rotated $0^{\circ}-360^{\circ}$ in $15^{\circ}$ steps.

The URA signals showed low modulation (less then $1 \%$ for $100 \%$ input), as in the example of Figure 10 . The DoLP (Table 7) is very low (better) than the requirement. The low amplitude indicates that the URA effectively scrambles highly polarized input light.

The trend of the URA DoLP was evaluated over the test period and environmental conditions, and showed no significant change. The primary purpose of the URA is to determine relative gain coefficients of detectors measuring orthogonal polarizations. Changes in these coefficients between measurements at Raytheon and with the APS installed in the Glory spacecraft were less than $0.1 \%$. Subsequent measurements of the relative gain coefficients using the URA during TVAC testing of the Glory spacecraft continued to be show stable behavior with less than $0.1 \%$ variations over the entire test.

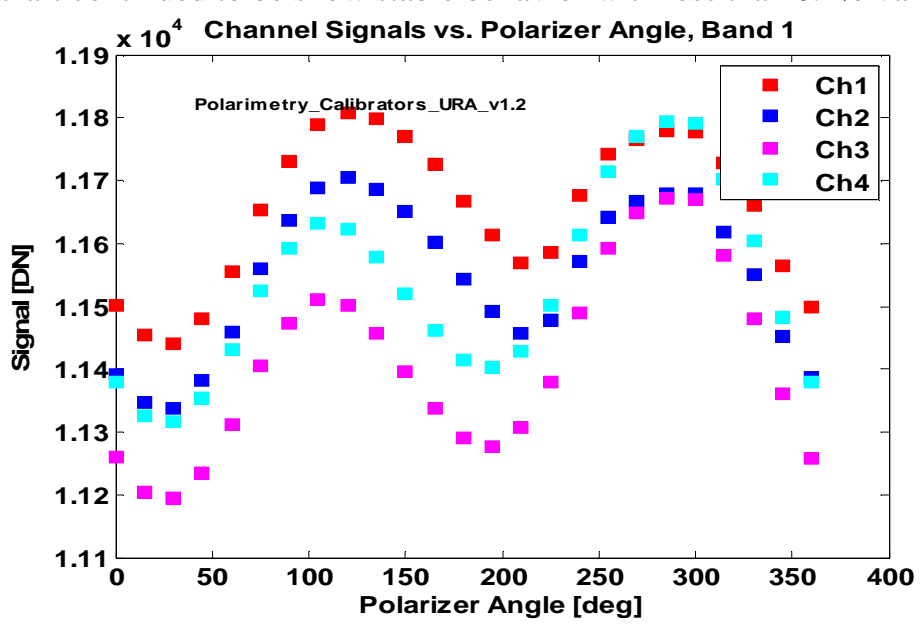

Figure 10: Example of the URA signal with a polarized source

Table 7: Calculated URA DoLP

\begin{tabular}{|c|c|}
\hline \multirow{2}{*}{ Band } & URA DOLP \\
\cline { 2 - 2 } & Reqt: $<0.05$ \\
\hline 1 & 0.003 \\
\hline 2 & 0.002 \\
\hline 3 & 0.001 \\
\hline 4 & 0.008 \\
\hline 5 & 0.001 \\
\hline 6 & 0.005 \\
\hline 7 & 0.006 \\
\hline 8 & 0.002 \\
\hline 9 & 0.007 \\
\hline
\end{tabular}




\subsection{Polarized Reference Assembly}

A second nadir-facing polarimetric calibrator, the Polarized Reference Assembly (PRA), contains a set of Glan-Taylor prisms for the VNIR bands and a wire grid polarizer for the SWIR bands to create a view for all channels that is highly polarized with a known azimuth. The PRA is used to ensure that the absolute degree of linear polarization of scene measurements is correct. It is also used to monitor and correct for any change in instrumental polarization that may occur over the mission lifetime.

The APS internal PRA was tested in a similar procedure to that of measuring the URA. The external polarized and unpolarized sources were used to determine the exact orientation of the Glan-Taylor and wire-grid polarizers in the PRA. This allows the depolarization coefficients $\alpha_{\mathrm{q}}$ and $\alpha_{\mathrm{u}}$ to be obtained from observations of the PRA. The Glan-Taylor polarizers used to calibrate telescope pairs in the VNIR are not perfectly aligned with $90^{\circ}$ between their polarization axes (mis-alignments are $1^{\circ}$ for A-telescopes and $1.5^{\circ}$ for B-telescopes) and the DoLP observed looking at the two different polarizers is not therefore expected to be $100 \%$, and can indeed be greater than $100 \%$. Since the wire grid polarizer used in the PRA covers both telescopes SWIR, the DoLP observed by the SWIR telescopes should be close to 100\%, and is, to within $0.03 \%$. This behavior of the PRA for the VNIR bands was expected and is the reason for the DoLP values shown in Table 8. In fact, the primary measure of the PRA performance is the comparison of the depolarization coefficients $\alpha_{q}$ and $\alpha_{u}$ determined from a complete polarimetric calibration and those obtained from the PRA that agreed with one another over TVAC thermal environments to within $0.25 \%$. This measure continued to be stable to within $0.2 \%$ during TVAC testing of APS on the spacecraft.

Table 8: The DoLP of the PRA shows complete polarization

\begin{tabular}{|c|c|c|c|}
\hline \multirow{2}{*}{ Band } & PRA DOLP & \multirow{2}{*}{ Stokes q } & Stokes u \\
\cline { 2 - 4 } & Req't: $>0.99$ & & \\
\hline 1 & 1.011 & -0.706 & 0.723 \\
\hline 2 & 1.004 & -0.699 & 0.721 \\
\hline 3 & 1.011 & -0.707 & 0.723 \\
\hline 4 & 1.004 & -0.699 & 0.721 \\
\hline 5 & 1.011 & -0.706 & 0.723 \\
\hline 6 & 1.004 & -0.699 & 0.720 \\
\hline 7 & 0.999 & 0.688 & -0.724 \\
\hline 8 & 0.999 & 0.687 & -0.724 \\
\hline 9 & 0.999 & 0.688 & -0.725 \\
\hline
\end{tabular}

Trending of the PRA parameters showed that they were consistent with the baseline results. The Stokes parameters were consistent within $0.3 \%$, and the DoLP within $0.4 \%$. The polarization angles of the PRA were consistent within $0.14^{\circ}$.

\subsection{Dark Reference Assembly}

The Dark Reference Assembly (DRA) is designed to extinguish stray light that is incident upon it. Tests were performed to characterize possible leakage of radiance into the DRA when the APS was viewing a bright scene (1000 W lamp). The maximum change in the APS Dark Reference signal did not exceed the maximum allowable $0.05 \%$ of scene radiance (APS requirement), when compared to a dark reference signal with zero scene radiance (Figure 11). 


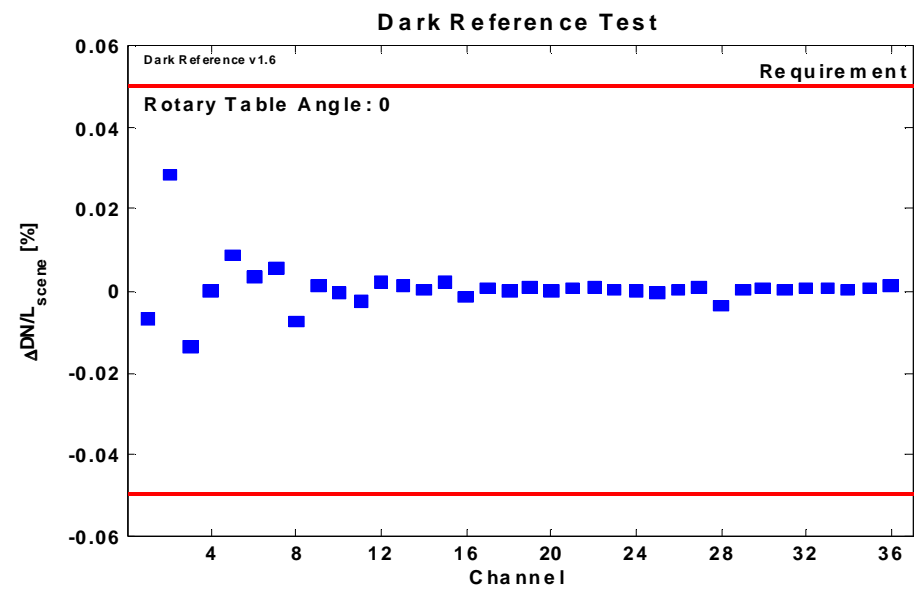

Figure 11: Maximum change in DRA signal

\subsection{Solar Reference Assembly}

The Solar Reference Assembly (SRA) provides for absolute radiometric correction by means of solar reflectance from a well characterized Spectralon ${ }^{6}$ diffuser plate. The bi-directional reflectance distribution function (BRDF) and surface flatness was characterized on the ground so that the angularly dependent reflectance of the diffuser will not be confused with the sun's angle of incidence variations, on orbit.

\section{Summary}

The Aerosol Polarimetry Sensor is the most accurate instrument ever built for measuring aerosol properties and distributions from space. The launch is planned for November, 2010. Its performance was thoroughly evaluated in ground testing, both at the instrument and at the system level. The tests evaluated all significant characteristics of the instrument, and focused on radiometric and polarimetric performance. The APS exceeded nearly all requirements with very substantial margins. All the key characteristics were evaluated before and after the environmental tests, particularly thermal-vacuum testing. The thorough evaluation of the trends of the key parameters showed minimal changes and high stability. The sensor performance is significantly better than required, which will lead to more accurate Environmental Data Records (EDRs). The APS will enable high accuracy modeling of the aerosols in the atmosphere, leading to more effective analysis of global warming.

\section{ACKNOWLEDGEMENTS}

The APS project has been funded by NASA. The authors thank the community of people dedicated to advancing the study of global climate change and its implications. We acknowledge in particular the work of the NASA consultants Dr. Edgar E. Russell and Richard A. Chandos who were instrumental in the successful completion of the APS sensor.

\section{REFERENCES}

[1] GSFC overview: http://glory.gsfc.nasa.gov/overview-aps.html

[2] Richard Peralta et al., "Aerosol Polarimetry Sensor for the Glory Mission" Proc. SPIE 6786 (2007).

[3] M. Mishchenko et al., "Accurate Monitoring of Terrestrial Aerosols and Total Solar Irradiance" BAMS 88, 677 (2007).

[4] Butler, James J. et al., "Radiometric Measurement Comparison on the Integrating Sphere Source Used to Calibrate the Moderate Resolution Imaging Spectroradiometer (MODIS) and the Landsat 7 Enhanced Thematic Mapper Plus (ETM+)", Journal of Research of the National Institute of Standards and Technology, Vol. 108, Number 3, (2003).

[5] B. Cairns et al., "The Research Scanning Polarimeter - Calibration and Ground-based measurements" Proc SPIE 3754 (1999).

[6] G. T. Georgiev, et al., "BRDF Study of Gray-Scale SPECTRALON" Proc SPIE 7081, (2008). 